\title{
Type A microsatellite instability in pediatric gliomas as an indicator of Turcot syndrome
}

\author{
Laura Giunti ${ }^{1}$, Valentina Cetica ${ }^{2}$, Ugo Ricci $^{1}$, Sabrina Giglio ${ }^{1,3}$, Iacopo Sardi ${ }^{2}$, \\ Milena Paglierani ${ }^{4}$, Elena Andreucci ${ }^{1}$, Massimiliano Sanzo ${ }^{5}$, Marco Forni $^{6}$, \\ Anna Maria Buccoliero ${ }^{4}$, Lorenzo Genitori ${ }^{5}$ and Maurizio Genuardi*,1,3,7
}

\begin{abstract}
${ }^{1}$ Medical Genetics Unit, Meyer Children's University Hospital, Florence, Italy; ${ }^{2}$ Department of Pediatrics, University of Florence, Florence, Italy; ${ }^{3}$ Department of Clinical Pathophysiology, University of Florence, Florence, Italy; ${ }^{4}$ Department of Human Pathology and Oncology, Careggi University Hospital, Florence, Italy; ${ }^{5}$ Neurosurgery Unit, Meyer Children's University Hospital, Florence, Italy; ${ }^{6}$ Pathology Department, Regina Margherita Children's Hospital, Turin, Italy; ${ }^{7}$ Fiorgen Foundation for Pharmacogenomics, Sesto Fiorentino, Italy
\end{abstract}

Microsatellite instability (MSI) is present in hereditary conditions due to mismatch repair (MMR) gene mutations. Following MSI analysis, tumor samples are classified into MSS (stable), MSI-L (low instability), and MSI-H (high instability) based on the fraction of unstable loci. Another MSI-based classification takes into account the size difference between mutant alleles in tumor DNA compared to wild-type alleles; two types of MSI, A and B, are recognized using this approach, type $A$ being characterized by smaller, more subtle allelic shifts compared to type B. Biallelic mutations of MMR genes are associated with pediatric cancers, including glial tumors, in Turcot syndrome type 1 (TS1). However, most TS1-associated gliomas so far analyzed did not display MSI. We investigated the frequency of MSI in a series of 34 pediatric gliomas of different grade using a panel of five mononucleotide quasimonomorphic markers. Subtle qualitative changes were observed for the majority of markers in two glioblastomas (5.9\% of the total series and $33.3 \%$ of glioblastomas). In both cases, family histories were compatible with TS1, and mutations of the PMS2 and MLH1 genes were identified. In one family, the MSI patterns were compared between the glioblastoma and a colon cancer from an affected relative, showing a clear qualitative difference, with the former displaying type $A$ and the latter type B instability, respectively. These results were confirmed using additional microsatellite markers, indicating that knowledge of the association between TS1-related glial tumors and subtle type A MSI is important for full ascertainment of TS1 patients and appropriate counselling.

European Journal of Human Genetics (2009) 17, 919-927; doi:10.1038/ejhg.2008.271; published online 21 January 2009

Keywords: Lynch syndrome; hereditary cancer; mismatch repair; PMS2; MLH1

\section{Introduction}

Turcot syndrome is a genetically heterogeneous condition defined by the association of colorectal and central

*Correspondence: Professor M Genuardi, Section of Medical Genetics, Department of Clinical Pathophysiology, University of Florence, Viale Pieraccini 6, 50139 Florence, Italy.

Tel: + 39-055-4271-421; Fax: + 39-055-4271-413;

E-mail: m.genuardi@dfc.unifi.it

Received 5 August 2008; revised 23 October 2008; accepted 17 December 2008; published online 21 January 2009 nervous system (CNS) tumors. ${ }^{1}$ Two forms of Turcot syndrome are recognized based on the type of CNS tumor and on the underlying molecular defect. Type 1 (TS1) is characterized by the occurrence of glial tumors and is caused by mutations of any of the four mismatch repair (MMR) genes (MLH1, MSH2, PMS2 and MSH6) involved in cancer susceptibility. ${ }^{2,3}$ Heterozygous mutations at an MMR locus are associated with Lynch syndrome. ${ }^{4,5}$ On the other hand, individuals who have both copies of an MMR gene mutated develop a very severe condition with 
childhood cancers and manifestations of neurofibromatosis 1 (NF1). ${ }^{2,3}$ Type 2 Turcot syndrome is a distinct entity caused by mutations of the APC gene, whose phenotypic manifestations are intestinal polyposis, colorectal carcinoma (CRC) and medulloblastoma. ${ }^{4,5}$

An important step in the diagnosis of inherited conditions due to defective MMR function is represented by the detection of heterogeneous somatic length alterations of short repeated DNA sequences, or microsatellites, in tumor DNA. Testing for microsatellite instability (MSI) is accomplished by investigation of multiple microsatellite markers in tumor DNA. ${ }^{2}$ Tumors are classified into three categories according to the proportions of markers showing instability: MSI-H (high-level MSI), MSI-L (low-level MSI) and MSS (microsatellite stable), which have $\geq 30-40 \%,>0 \%-<30-40 \%$, and $0 \%$ unstable markers, respectively. ${ }^{6}$ A distinct classification considers the size of the insertion/deletions events, rather than the number of altered loci; using this system, unstable samples can be divided into type A and type B, with the former displaying smaller allelic shifts compared to the latter. ${ }^{7,8}$

The prevalence of MSI in unselected pediatric and/or adult glial cell tumors ranges from 0 to $33 \% .^{9-15}$ The majority of gliomas from TS1 patients investigated so far were MSS. ${ }^{16-19}$ Such variable findings could be partly explained by different sensitivities of the microsatellite panels used for MSI evaluation.

The original microsatellite panel proposed for the investigation of MSI in colorectal cancer included two mononucleotide (BAT25 and BAT26) and three dinucleotide (D2S123, D5S346, and D17S250) repeats. ${ }^{6}$ Mononucleotide tracts, especially $B A T 26$, were found to be the most sensitive markers of MSI, and it was proposed that BAT26 be used alone to identify colorectal cancers associated with Lynch syndrome. ${ }^{20}$ However, interethnic variation in the number of repeats, ${ }^{21}$ and the not infrequent occurrence of homozygous deletions of the region containing the BAT26 poly-A repeat in cancers from patients heterozygous for MSH2 mutations, ${ }^{22}$ indicate that a proportion of Lynch syndrome cancers might be missed with this approach.

Therefore, further panels comprised of five different mononucleotide repeats have been proposed for MSI analysis. ${ }^{21,23}$ These mononucleotide markers are defined as quasimonomorphic because, although they are polymorphic in the population due to the existence of variable numbers of repeat units, interallelic size differences are very small for each single locus. This property allows easy and reliable detection of the dramatic size shifts characteristic of type B MSI without the need to investigate constitutional DNA from the same patient for comparison. ${ }^{23}$ In addition, it has been proposed that, using these panels, type A MSI may be revealed by the presence of allelic variants that are rare in the general population at $\geq 3$ loci when only tumor DNA is investigated. ${ }^{21}$
In this work, we employed a five mononucleotide marker panel to investigate MSI in a series of 34 pediatric glial tumors, to establish the frequency and patterns of MSI and its correlation with cancer family history. Two positive cases, both from TS1 families, were identified. Both of them showed allelic shifts of reduced size compared to the usual findings in Lynch syndrome associated CRCs.

\section{Materials and methods \\ Patients and samples}

Patients Patients were ascertained through the Neurosurgery Unit of the Meyer Children's University Hospital, Florence, in the period March 2004-October 2006. Overall, samples from 34 different patients were analyzed, including twenty-eight low-grade and six high-grade tumors according to the WHO classification (Table 1). ${ }^{24}$ Eighteen samples were collected in liquid nitrogen immediately upon surgery. Paraffin-embedded specimens were obtained from patients for whom fresh-frozen samples were not available. To compare the results obtained on frozen and paraffin-embedded samples, paraffin blocks were retrieved and analyzed for six cases in the series of frozen tumors. Age at diagnosis ranged from 5 months to 14 years and 7 months (mean 6.9 years). All tumor samples were re-evaluated by a pathologist (AMB) before DNA extraction, and were judged to contain $>80 \%$ tumor cells. Peripheral blood for comparison between tumor and normal DNA was obtained by venipuncture from two patients. Further samples were obtained from the parents (blood) and sister (blood and fresh-frozen tumor and normal colonic mucosa) of case 121. Informed consent was obtained from all study participants.

\section{DNA extraction}

DNA was extracted from peripheral blood and frozen tumors using the QIAamp DNA Mini Kit (Qiagen, Milan, Italy) and from paraffin-embedded specimens using a manual protocol. ${ }^{25}$ DNA concentrations were determined using the Quantifiler ${ }^{\mathrm{m} /}$ Human DNA Quantification Kit (Applied Biosystems, Foster City, CA, USA).

\section{Microsatellite analysis}

The mononucleotide markers BAT25, BAT26, NR21, NR22 and NR24 were amplified with a multiplex PCR proto$\mathrm{col}^{21,23}$ using fluorescently labelled primers and $2 \mathrm{ng}$ of genomic DNA as template. Products were run on a 310 ABI PRISM Genetic Analyzer (Applied Biosystems, Foster City, CA, USA) and analyzed with GeneScan ${ }^{\circledR}$ and Genotyper ${ }^{\circledR}$ softwares (Applied Biosystems, Foster City, CA, USA). PCR conditions included an initial denaturation of $15^{\prime}$ at $95^{\circ} \mathrm{C}$, followed by 29 amplification cycles $\left(30^{\prime \prime}\right.$ at $94^{\circ} \mathrm{C}, 90^{\prime \prime}$ at $55^{\circ} \mathrm{C}$ and $60^{\prime \prime}$ at $72^{\circ} \mathrm{C}$ ), and by a final extension step of $30^{\prime}$ at $60^{\circ} \mathrm{C}$. 
Table 1 Clinical characteristics of the 34 gliomas and genotypes at quasimonomorphic mononucleotide repeats

\begin{tabular}{|c|c|c|c|c|c|c|c|}
\hline \multirow[b]{2}{*}{ Sample ID } & \multirow[b]{2}{*}{ Age at diagnosis } & \multirow[b]{2}{*}{ Histology ${ }^{\mathrm{a}}$} & \multicolumn{5}{|c|}{ Genotypes $^{\mathrm{b}}$} \\
\hline & & & BAT 25 & $B A T 26$ & $N R 21$ & $N R 22$ & $N R 24$ \\
\hline 1 & 10 years and 5 months & $X A$ & $120-121$ & $118-118$ & $109-110$ & $138-138$ & $128-128$ \\
\hline 6 & 13 years and 9 months & PA & $122-122$ & $117-117$ & 109-109 & $137-138$ & $127-128$ \\
\hline 13 & 7 years and 8 months & XA & $121-122$ & $117-117$ & $110-111$ & $137-138$ & $127-128$ \\
\hline 15 & 9 years and 11 months & PA & $123-123$ & $117-117$ & $109-111$ & $137-138$ & $128-128$ \\
\hline 18 & 14 years and 7 months & PA & $123-123$ & $117-117$ & $110-110$ & $137-138$ & $127-128$ \\
\hline 36 & 5 years and 6 months & PA & $122-122$ & $117-118$ & 109-109 & $137-138$ & $128-128$ \\
\hline 40 & 12 years and 3 months & PA & $123-124$ & $117-117$ & $109-110$ & $137-138$ & $128-130$ \\
\hline 43 & 1 year and 9 months & FA & $122-122$ & $117-117$ & $110-110$ & $137-138$ & $128-128$ \\
\hline 84 & 14 years and 7 months & GBM & $124-124$ & $117-117$ & n.a. & $137-138$ & $128-128$ \\
\hline 98 & 4 years and 2 months & GBM & $122-122$ & $117-117$ & $110-110$ & $137-137$ & $128-128$ \\
\hline 108 & 6 years and 9 months & PA & $122-124$ & $117-117$ & $110-110$ & $137-138$ & $128-128$ \\
\hline 110 & 11 years and 3 months & PA & $123-124$ & $116-117$ & $110-110$ & $137-138$ & $128-128$ \\
\hline 111 & 2 years and 7 months & PA & $121-122$ & $117-117$ & $105-110$ & $137-138$ & $128-128$ \\
\hline 112 & 2 years and 3 months & PA & $121-121$ & $115-117$ & $110-111$ & $137-138$ & $127-128$ \\
\hline 113 & 1 year and 2 months & PA & $122-123$ & $117-117$ & $109-110$ & $137-137$ & $128-129$ \\
\hline 114 & 0 year and 10 months & PA & $122-123$ & $117-117$ & $110-110$ & 137-137 & $128-128$ \\
\hline 115 & 0 year and 5 months & PA & $122-122$ & $117-117$ & $110-111$ & $137-138$ & $128-128$ \\
\hline 116 & 7 years and 4 months & PA & $122-123$ & $117-117$ & $109-110$ & $138-138$ & $127-128$ \\
\hline 117 & 3 years and 5 months & PA & $121-122$ & $117-117$ & $110-110$ & $137-138$ & $128-128$ \\
\hline 118 & 7 years and 3 months & PA & $122-122$ & $117-117$ & $109-110$ & $137-138$ & $128-129$ \\
\hline 119 & 1 year and 5 months & PA & $121-123$ & $115-117$ & $110-110$ & 137-137 & $128-128$ \\
\hline 120 & 3 years and 5 months & PA & $121-121$ & $117-117$ & $109-110$ & $137-138$ & $128-128$ \\
\hline 121 & 10 years and 3 months & GBM & MSI & MSI & MSI & n.a. & n.a. \\
\hline 122 & 6 years and 2 months & PA & $120-123$ & $117-118$ & $109-109$ & $137-138$ & $128-128$ \\
\hline 123 & 12 years and 3 months & PA & $121-123$ & $117-118$ & $110-110$ & 137-137 & $127-128$ \\
\hline 124 & 14 years and 6 months & FA & $122-122$ & $117-118$ & 109-110 & $137-138$ & $128-128$ \\
\hline 127 & 7 years and 3 months & PA & $121-122$ & $117-117$ & $110-110$ & $137-137$ & $128-128$ \\
\hline 132 & 3 years and 9 months & FA & $122-124$ & $117-117$ & $110-110$ & $138-138$ & $128-129$ \\
\hline 135 & 11 years and 0 month & PA & $122-122$ & $118-118$ & $109-110$ & $137-138$ & $128-129$ \\
\hline 139 & 8 years and 5 months & FA & $122-124$ & $118-118$ & $110-110$ & $137-138$ & $127-128$ \\
\hline 143 & 4 years and 7 months & PA & $121-122$ & $118-118$ & $110-110$ & 137-137 & $128-128$ \\
\hline 151 & 2 years and 0 month & GBM & $122-122$ & $117-117$ & $109-110$ & $137-137$ & $128-128$ \\
\hline & 4 years and 0 months & GBM & $122-122$ & $117-117$ & $110-110$ & $137-138$ & $128-128$ \\
\hline & 4 years and 10 months & GBM & $121-122$ & MSI (113-117) & MSI (106-109) & MSI (136-137) & $127-128$ \\
\hline
\end{tabular}

${ }^{a}$ XA: xantoastrocytoma; FA: fibrillary astrocytoma; PA: pilocytic astrocytoma; GBM: glioblastoma multiforme.

bold: rare alleles not observed in a control group; MSI: microsatellite instability; n.a.: not amplified.

The mononucleotide repeats BAT40 and NR27 and the dinucleotide repeats D2S123, D5S346 and D17S250 were amplified individually using published primer sequences. ${ }^{6,21}$

\section{Immunohistochemical analysis of MMR proteins}

For each lesion, representative $4 \mu \mathrm{m}$-thick sections were cut from tissue blocks of formalin-fixed, paraffin-embedded tissues. Slides were deparaffinized in Bio-Clear (Bio-Optica, Milan, Italy) and hydrated with graded ethanol concentrations. Tissue sections were treated with mouse anti-MLH1, clone G168-728 (Cell Marque, Hot Springs, AR, USA), mouse anti-MSH2, clone G219-1129 (Cell Marque), mouse anti-MSH6, clone 44 (BD Biosciencies, Pharmingen, San Diego, CA, USA) and mouse-PMS2, clone A16-4 (BD Biosciencies) monoclonal antibodies.

MLH1 and MSH2 expression was evaluated using the iVIEW DAB Detection Kit (Ventana) as revelation system, whereas immunohistochemical analyses of MSH6 and PMS2 were performed using a manual protocol.

\section{MMR gene analysis}

The complete coding sequence and flanking exon-intron borders of the MLH1 and PMS2 genes were investigated by direct sequencing. The PMS2 sequence was investigated using a nested PCR approach ${ }^{26}$ to avoid amplification of related pseudogene sequences. The presence of MLH1 genomic rearrangements and the methylation status of the MLH1 promoter were investigated by multiplex ligation-dependent probe amplification (MLPA), using the kits P003 MLH1/MSH2 probemix and ME001 Tumor Suppressor-1, respectively (MRC Holland, Amsterdam, Netherlands) according to the manufacturer's instructions. Mutation nomenclature follows the Human Genome Variation Society (URL: http://www.hgvs.org/mutnomen/) recommendations. DNA mutation numbering is based on the PMS2 and MLH1 cDNA sequences (GenBank accession 
numbers NM_000535.4 and NM_000249.2, respectively) with the A of the ATG translation-initiation codon numbered as +1 . Amino acid numbering starts with the translation initiator methionine as +1 .

\section{Results \\ MSI analysis with quasimonomorphic mononucleotide markers in glioma samples}

Of the 34 glioma DNA samples, thirty-one did not show apparent instability at any of the five mononucleotide markers (Table 1). For six of these tumors (samples 1, 6, 15, 43, 98 and 153), DNA was extracted from both frozen and paraffin-embedded specimens, obtaining identical electrophoretic profiles. All mononucleotide marker alleles present in this group had been observed in a sample of 112 cancer-free control individuals from the same geographic area (data not shown). Leukocyte DNA was available for comparison from two patients (111 and 139) in this group, and no differences were observed between the DNA profiles of tumor and normal samples.

One sample (number 40), a low grade pilocytic astrocytoma, showed a single $130 \mathrm{bp}$ allele at the NR24 locus (Table 1) that had not been detected in other tumors investigated in this study nor in control subjects (data not shown). This allele is likely a rare subpolymorphic variant, whose occurrence was previously documented at noncoding mononucleotide repeat loci. ${ }^{21}$

Thus, the vast majority of the samples evaluated (32/34 in the whole series) were MSS. In the remaining two cases (121 and 153), amplification profiles at several loci were indicative of MSI (Table 1; Figures 1-2). In sample 121, BAT25, BAT26 and NR21 showed an unstable profile, with presence of multiple peaks, whereas NR22 and NR24 amplification products were consistently below the detection threshold (Figure 1). The lack of NR22 and NR24 PCR products could be related to the quality of the sample or to homozygous deletions of the chromosomal regions where these markers are located.

Comparison of microsatellite profiles between normal brain and tumor DNA from patient 153 revealed different patterns at 4/5 loci (Figure 2). Allelic size differences between normal and tumor DNA were limited to two (113 vs $115 \mathrm{bp}$ at BAT26; 106 vs $108 \mathrm{bp}$ at NR21) or only $1 \mathrm{bp}$ (BAT25 and NR22). Interestingly, the tumor profiles of markers BAT26, NR21 and NR22 had the appearance of heterozygous genotypes carrying a rare allele, not detected in any other tumor or control sample (Table 1 and Figure 2). Both frozen and paraffin-embedded tumor DNA samples were investigated for this case, yielding identical results.

\section{Clinical and family histories of the two patients with MSI-H gliomas}

Both samples 121 and 153 were high-grade gliomas (glioblastomas). Ages at diagnosis were 10 years, 3 months and 4 years, 10 months for patients 121 and 153, respectively. The presence of multiple cafè-au-lait spots had initially raised a suspicion of NF1 in patient 121 .

Patent 121 was the second born of three siblings. His younger sister died at the age of 4 years due to a brainstem malignancy of undetermined type. The elder sister (121S) was diagnosed with mucinous carcinoma of the ascending colon at the age of 21 years. Parents were healthy and unrelated, and family history was otherwise unremarkable, except for a maternal second cousin, who had died of leukemia at the age of 11 years, and his mother, who had a diagnosis of colorectal cancer at 28 years (Figure 3 ).

The sister and father of patient 153 had died of glioblastoma and pancreatic cancer at the ages of 12 and 50 years, respectively; no other details on family history were available for this case.

Therefore, family histories of both patient 121 and 153 suggested a diagnosis of TS1. No other patient had a history of pediatric tumors among siblings.

Comparative MSI analysis in the family of patient 121 Availability of samples from relatives of patient 121 allowed us to compare microsatellite electrophoretic profiles in different normal and tumor specimens (Figure 1). The glioblastoma showed peaks that were not found in parental DNA for BAT25, BAT26 and NR21, confirming the presence of MSI.

The pattern of instability in the glioblastoma from patient 121 and in his sister's colon cancer was clearly different (Figure 1). The colon cancer displayed type B instability at 4/5 (BAT25, BAT26, NR21, and NR22) loci, as shown by the appearance of distinct sets of peaks of shorter molecular weight compared to constitutional alleles. In addition, the heights of the latter were remarkably reduced compared to normal tissues (Figure 1). On the other hand, the profiles of the three markers amplified from the glioblastoma showed a continuous peak distribution.

\section{MSI analysis of additional microsatellites in MSI-H glioma samples}

Further markers were investigated in the two samples showing MSI. BAT40, a highly polymorphic mononucleotide marker, was unstable in both samples, but the size of the shifts was lower in the glioblastomas compared to the colon cancer from patient 121S (Figure 4). Similarly, small (2 bp) size shifts were observed in sample 153 at the dinucleotide markers D2S123 and D17S250, whereas the difference between mutant and constitutional alleles was much larger (6-8 repeats) in the colon cancer from patient $121 \mathrm{~S}$ (data not shown). The dinucleotide marker D5S346 was stable in the two glioblastomas and in the colon cancer from patient $121 \mathrm{~S}$, whereas the quasimonomorphic marker NR27 showed type B MSI in the colon cancer sample but was stable in the glioblastomas (data not shown). No 
BAT25
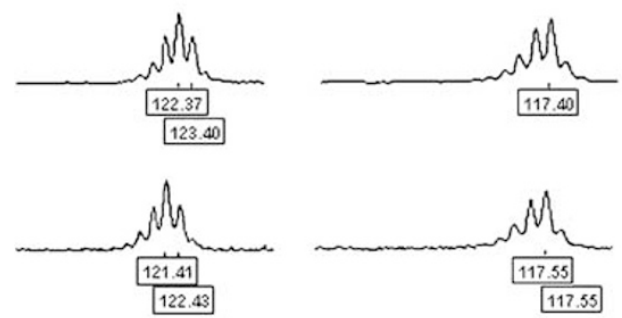
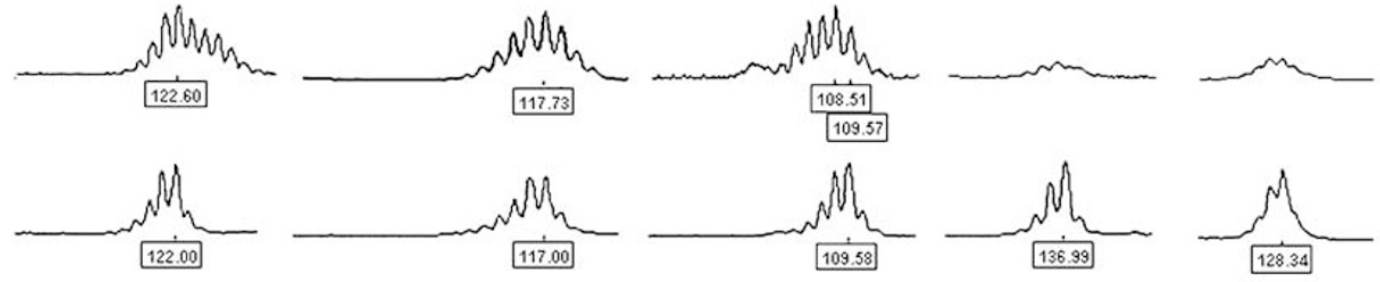

121S-bl
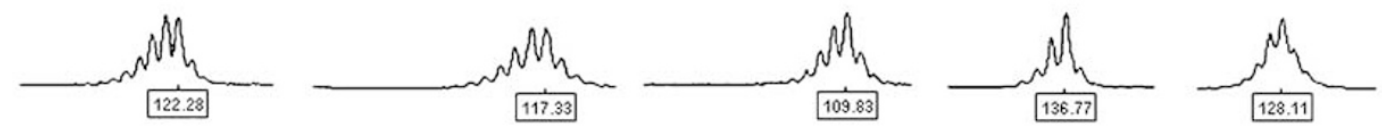

121S-nm
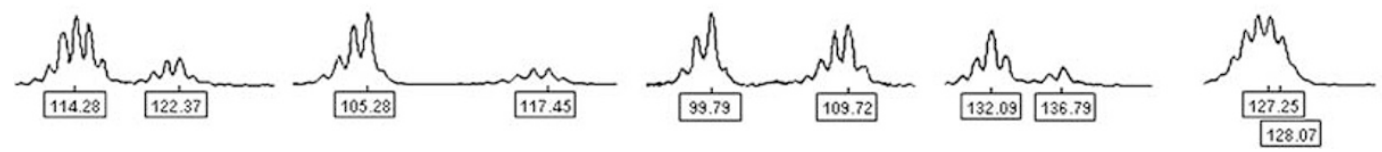

121S-t

Figure $1 \mathrm{MSI}$ analysis with quasimonomorphic mononucleotide markers in family 121. 121F: father, peripheral leukocytes; $121 \mathrm{M}$ : mother, peripheral leukocytes; 121: proband, glioblastoma; 121S-bl: sister, peripheral leukocytes; 121S-nm: sister, normal intestinal mucosa; 121S-t: sister, colon cancer. Numbers at the bottom of each electropherogram indicate size (in bp).

BAT25

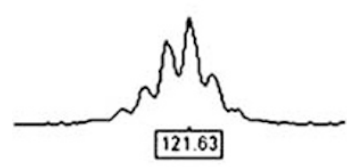

121.63

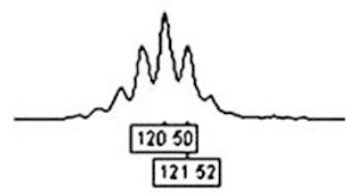

BAT26

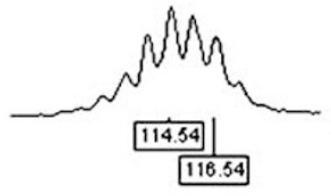

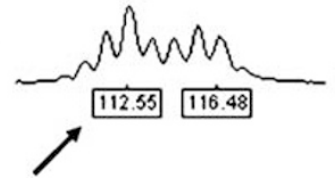

NR21
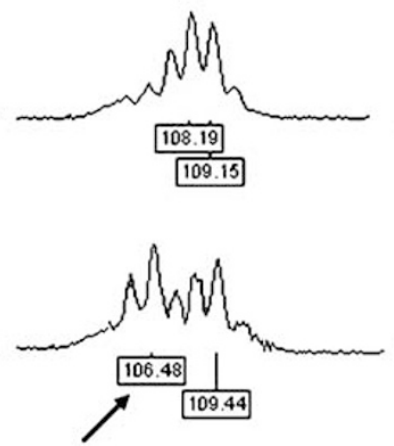

NR22

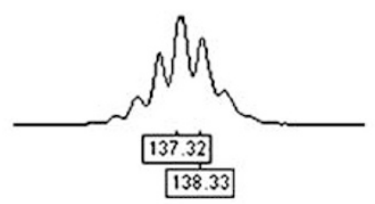

$153 \mathrm{~N}$

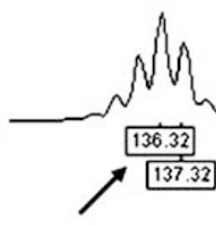

$153 T$

Figure $2 \mathrm{MSI}$ analysis with quasimonomorphic mononucleotide markers in normal (153N) and tumor (153T) DNA from patient 153. All markers shown are unstable in tumor DNA, as demonstrated by the presence of different profiles in tumor compared to normal DNA. Numbers at the bottom of each electropherogram indicate size (in bp). Arrows in tumor DNA indicate peaks corresponding to allelic sizes not present in a control population.

amplification product was observed for markers D2S123 and D17S250 in sample 121.

\section{Immunohistochemical analysis of MMR proteins} Immunohistochemical analysis of the MMR proteins was conducted on the glioblastoma from patient 153 and on the colon cancer from 121S, whereas no interpretable results could be obtained on the glioblastoma from case 121 . The colon cancer sample showed isolated lack of PMS2 expression, while absence of both MLH1 and PMS2 staining was observed in sample 153 (data not shown). 


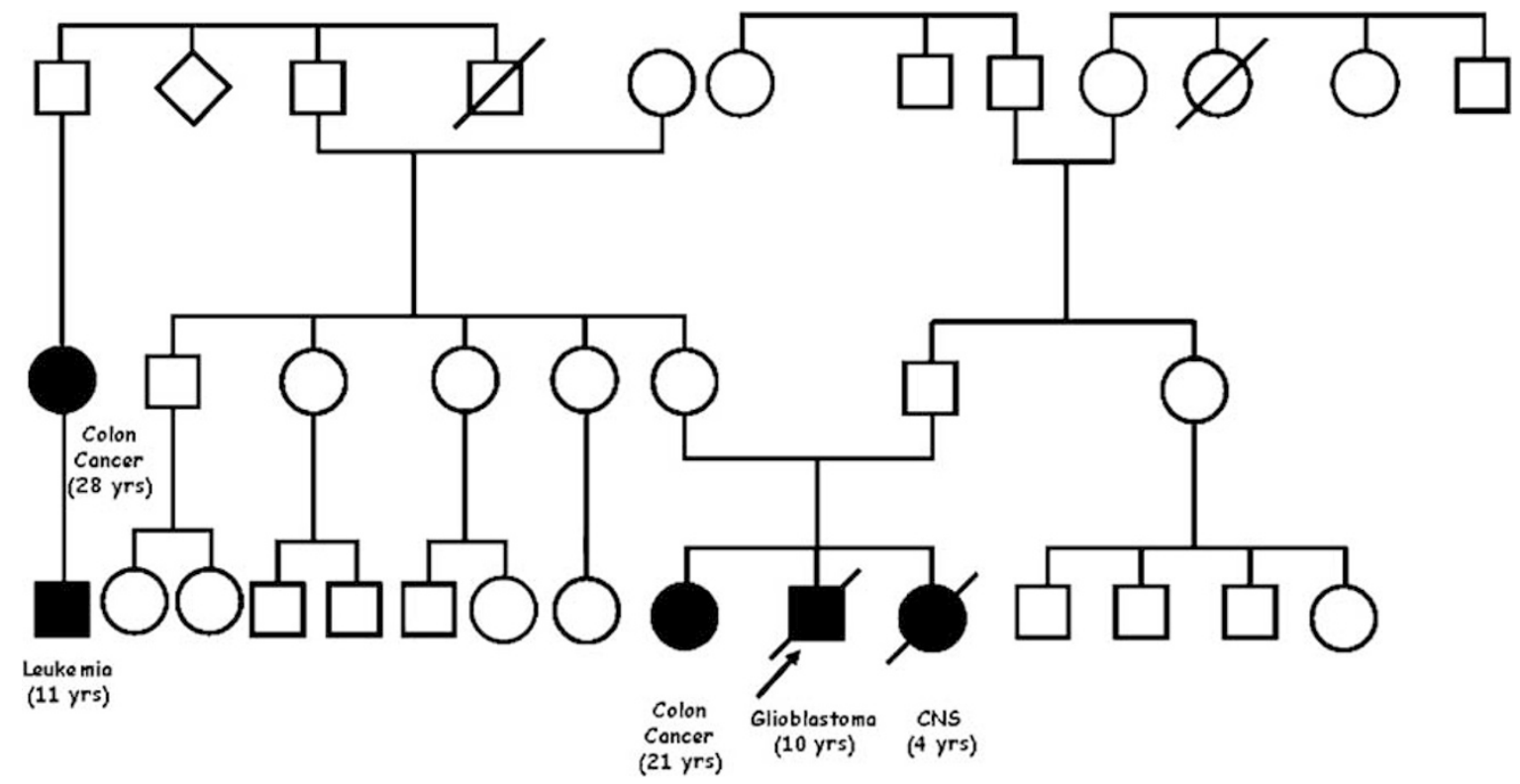

Figure 3 Pedigree of the family of patient 121. Ages at cancer diagnoses are shown.

\section{Mutation analysis of MMR genes}

Based on the immunohistochemistry results, mutation analysis of the PMS2 and MLH1 genes was undertaken in normal DNA from case $121 \mathrm{~S}$ and in case 153, respectively.

Two PMS2 single nucleotide substitutions, c.137G $>$ T and c.804-2A $>$ G, were identified in normal DNA samples from 121S. The former leads to the replacement of the aminoacid serine at position 46 with an isoleucine (p.Ser46Ile) in exon 2, whereas the latter affects the invariant AG dinucleotide of the exon 8 splice acceptor site. Both mutations were also present in the colon tumor of patient $121 \mathrm{~S}$ and in the brother's glioblastoma. The c. $137 \mathrm{G}>\mathrm{T}$ and c.804-2A $>\mathrm{G}$ mutations were inherited from the father and the mother, respectively.

A single MLH1 frameshift mutation, c.346_347insA, was identified in sample 153 by direct sequencing. No further MLH1 abnormality was detected by MLPA analysis of genomic rearrangements and promoter methylation.

\section{Discussion}

In this study, we have investigated the frequency of MSI in a series of pediatric gliomas using a panel of mononucleotide repeat markers. The percentage of MSI-H cases was $5.3 \%$ in the whole series, but substantially higher $(2 / 6$; $33.3 \%$ ) in high-grade glioblastomas, and both MSI-H samples were from patients with clinical and molecular characteristics of TS1.

The frequency of MSI was previously investigated in glial tumor series, with widely divergent results. Overall, data from previous studies agree on the finding that MSI is more common in pediatric high-grade gliomas. ${ }^{9-15,27-29}$ However, the proportion of MSI-H cases ranged from 0 to $33 \%$ in early-onset high-grade tumors among different studies. ${ }^{9,10,12-14}$ Variable microsatellite panels, with different sensitivity and specificity, have been used in these studies.

Interestingly, MSI has been detected in only 1 out of 6 gliomas from TS1 patients with biallelic MMR gene mutations investigated so far. ${ }^{17-19,30,31}$ It has been hypothesized that MMR gene defects may contribute to brain tumorigenesis through mechanisms other than canonical lack of replication error repair. ${ }^{18}$ It is also possible that MSI may be difficult to detect in CNS tumors due to a lower degree of instability, associated with smaller allelic shifts, than in colorectal tumors. The latter hypothesis finds some support from the observation that allelic shifts at microsatellite loci are less pronounced in MSI-H endometrial $v s$ colorectal tumors, ${ }^{32}$ which indicates that the entity of MSI varies among tumors of different histological derivation.

The degree of instability was subtle in the two MSI-H samples identified in this study. The size of alleles generated by somatic mutation in the glioblastoma from patient 121 was closer to that of constitutional alleles compared to the colorectal cancer from his sister. This gave rise to a continuous distribution of electrophoretic peaks in the glioblastoma, whereas the colon cancer displayed two separate sets of peaks in distinct size ranges, corresponding to wild-type and mutant alleles, respectively.

In case 153, the size shifts between constitutional and tumor DNA at quasimonomorphic markers were very small, with differences in the range of 1-2 bp. Although the glioblastoma did not show the multiple peaks usually 


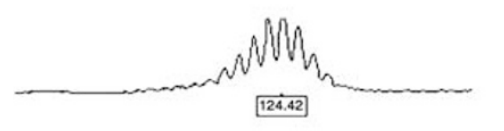

$153 N$

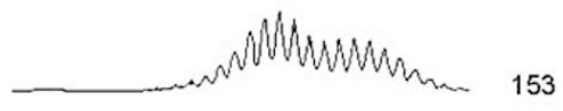

153

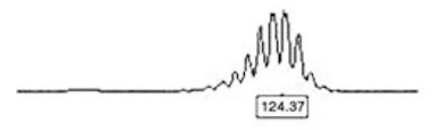

$121 \mathrm{~F}$

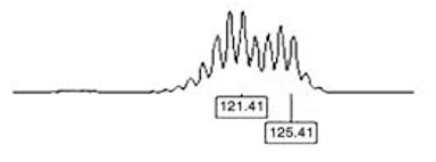

$121 \mathrm{M}$

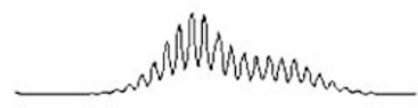

121

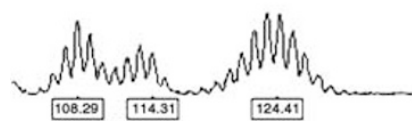

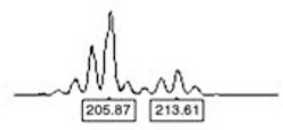

$153 N$

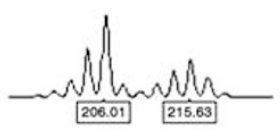

153
D17S250

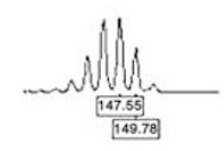

$153 \mathrm{~N}$

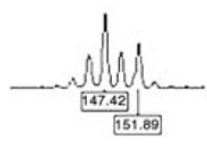

Figure 4 Type A MSI at the polymorphic mononucleotide repeat marker BAT40 (cases 153 and 121) and at dinucleotide markers D2S123 and D17S250 (case 153). 153N: proband from family 153, normal brain tissue; 153: proband from family 153, glioblastoma; $121 \mathrm{~F}$ : father, peripheral leukocytes; $121 \mathrm{M}$ : mother, peripheral leukocytes; 121 : proband from family 121 , glioblastoma; $121 \mathrm{~S}$-t: sister, colon cancer. Numbers at the bottom of each electropherogram indicate size (in bp).

associated with MSI, the electrophoretic profiles at 3/5 loci were compatible with heterozygous genotypes in the presence of rare alleles. As the occurrence of rare (frequency $<0.01$ ) constitutional alleles at $\geq 3$ quasimonomorphic mononucleotide loci is highly unlikely in individuals of Caucasian descent, it has been proposed that tumor samples showing this pattern be classified as MSI-H, even when normal matching DNA is not available for analysis. ${ }^{21}$ Therefore, this sample would have been considered MSI-positive according to this classification.

Although the commonly used definition of MSI differentiates samples based on the proportion of unstable loci, ${ }^{6}$ two qualitatively distinct types of MSI have been recognized according to the extent of allelic shifts compared with wild-type alleles at dinucleotide repeats. ${ }^{7,8}$ Type A MSI is characterized by dinucleotide shifts $\leq 6 \mathrm{bp}$, whereas type B MSI is associated with more dramatic changes. ${ }^{8}$ Human colorectal cancer samples usually display type B MSI, such as observed in the tumor sample from patient $121 \mathrm{~S}$. Instead, the pattern of MSI observed with the highly sensitive quasimonomorphic markers in the two glioblastomas in this series was of type A. Similar findings were observed for additional markers investigated. As glial cells presumably undergo a lower number of cell divisions than intestinal cells during the tumorigenic process, the higher degree of MSI in colon cancers could be the result of stronger somatic selection in favor of unstable alleles. Additional biological factors, such as tissue-specific redundancy of repair mechanisms, could also account for these differences.

The two patients with MSI-H gliomas in this series were from TS1 families, and in both cases mutations in PMS2 and $M L H 1$ were detected. The MLH1 frameshift mutation and the PMS2 splice mutation are clearly deleterious for protein function. The PMS2 missense mutation p.Ser46Ile has been previously identified in two unrelated TS1 families, from the United States and Italy, respectively. ${ }^{18,33}$ Although the clinical significance of missense mutations is often uncertain in the absence of functional studies, a pathogenic role for this variant was inferred based on evolutionary conservation of the involved aminoacid residue, non-conservative nature of the aminoacid change, and absence of the Ile46 variant in a control population. ${ }^{18}$

The finding of a single germ line mutation in patient 153 could be due to simple heterozygosity for an MLH1 mutation or to the presence of a second alteration not detectable despite extensive search with multiple methods, such as a deleterious change in regulatory or deep intronic regions. The presence of a sib affected with early-onset glioblastoma indicates that the latter hypothesis is more 
likely, as glial tumors occur at more advanced ages in heterozygotes for a single MMR gene mutation. ${ }^{2-4}$

The relationship between family history of cancer and MSI was previously evaluated in two studies of glioma patients, who reported the presence of MSI in 4/22 and 2/80 MSI-H high-grade tumors, respectively. ${ }^{10,12}$ Age at diagnosis ranged from 16 to 38 years, and 4/6 patients had a personal and/or family history of colorectal cancer. Monoallelic MSH2 or MLH1 germ line mutations were found in 5/6 cases, indicating a diagnosis of Lynch syndrome with CNS tumors. Alonso et al ${ }^{9}$ found $12 \mathrm{MSI}-\mathrm{H}$ out of 65 pediatric gliomas, and commented that it is unlikely that these could represent cases of TS, due to the rarity of this condition. However, the recent discovery that very early-onset cases of TS1 are associated with biallelic MMR gene mutations has been followed by an accrual in the number of reports of this condition, with $>30$ families described so far. ${ }^{3,31,34-37}$ Therefore, further investigations using sensitive microsatellite panels on larger series will be needed to determine the actual role of constitutional MMR gene defects in pediatric gliomas. However, as TS1 arises in individuals who are homozygotes or compound heterozygotes for MMR gene mutations, and the penetrance is reduced in simple heterozygotes, especially when PMS2 is involved, it has to be expected that a fraction of cases will have a negative cancer family history.

In conclusion, we found that a fraction of pediatric gliomas is associated with subtle, type A MSI in the context of TS1. Our observation that the degree of instability at each single locus is lower in MMR-related gliomas than in colorectal cancer might partially explain the discrepancies in MSI frequencies among different glioma series observed so far. Therefore, the use of a panel of at least five mononucleotide markers is recommendable for MSI analysis in glial tumors, especially when normal DNA is not available. These findings have important implications for the identification of TS1 and for clinical management of the patients and their relatives, as individuals with TS1 are at high risk of developing other tumor types, namely hematological malignancies and colorectal cancer, as well as other cancers observed in Lynch syndrome.

\section{Acknowledgements}

This work was supported by a PRIN grant of the Italian Ministry for University to MG and by contributions of Ente Cassa di Risparmio di Firenze to Fiorgen and MG. We are grateful to Roberta Sestini and Benedetta Toschi for assistance with MLPA analysis.

\section{References}

1 Turcot J, Despres JP, ST Pierre F: Malignant tumors of the central nervous system associated with familial polyposis of the colon: report of two cases. Dis Colon Rectum 1959; 2: 465-468.

2 Lucci-Cordisco E, Zito I, Gensini F, Genuardi M: Hereditary nonpolyposis colorectal cancer and related conditions. Am J Med Genet 2003; 122: 325-334.
3 Felton KEA, Gilchrist DM, Andrew SE: Constitutive deficiency in DNA mismatch repair. Clin Genet 2007; 71: 483-498.

4 Hamilton SR, Liu B, Parsons RE et al: The molecular basis of Turcot's syndrome. N Engl J Med 1995; 332: 839-847.

5 Paraf F, Jothy S, van Meir EG: Brain tumor-polyposis syndrome: two genetic diseases? J Clin Oncol 1997; 15: 2744-2758.

6 Boland CR, Thibodeau SN, Hamilton SR et al: A National Cancer Institute Workshop on microsatellite instability for cancer detection and familial predisposition: development of international criteria for the determination of microsatellite instability in colorectal cancer. Cancer Res 1998; 58: 5248-5257.

7 Thibodeau SN, Bren G, Schaid D: Microsatellite instability in cancer of the proximal colon. Science 1993; 260: 816-819.

8 Oda S, Mashara Y, Ikeda Y et al: Two modes of microsatellite instability in human cancer: differential connection of defective DNA mismatch repair to dinucleotide repeat instability. Nucleic Acids Res 2005; 33: 1628-1636.

9 Alonso M, Hamelin R, Kim M et al: Microsatellite instability occurs in distinct subtypes of pediatric but not adult central nervous system tumors. Cancer Res 2001; 61: 2124-2128.

10 Leung SY, Chan TL, Chung LP et al: Microsatellite instability and mutation of DNA mismatch repair genes in gliomas. Am J Pathol 1998; 153: $1181-1188$.

11 Cheng Y, Ng HK, Zhang SF et al: Genetic alterations in pediatric high-grade astrocytomas. Hum Pathol 1999; 30: 1284-1290.

12 Kanamori M, Kon H, Nobukuni T et al: Microsatellite instability and the PTEN1 gene mutation in a subset of early onset gliomas carrying germline mutation or promoter methylation of the hMLH1 gene. Oncogene 2000; 19: 1564-1571.

13 Szybka M, Bartkowiak J, Zakrzewski K, Polis L, Liberski P, Kordek R: Microsatellite instability and expression of DNA mismatch repair genes in malignant astrocytic tumors from adult and pediatric patients. Clin Neuropathol 2003; 22: 180-186.

14 Eckert A, Kloor M, Giersch A et al: Microsatellite instability in pediatric and adult high-grade gliomas. Brain Pathol 2007; 17: $146-150$.

15 Vladimirova V, Denkhaus D, Soerensen N, Wagner S, Wolff JE, Pietsch T: Low level microsatellite instability in paediatric malignant astrocytomas. Neuropathol Appl Neurobiol 2007; 34: 547-554.

16 Bougeard G, Charbonnier F, Moerman A et al: Early onset brain tumor and lymphoma in MSH2-deficient children. Am J Hum Genet 2003; 72: 213-216.

17 Menko FH, Kaspers GL, Meijer GA, Claes K, van Hagen JM, Gille JJ: A homozygous MSH6 mutation in a child with cafe-au-lait spots, oligodendroglioma and rectal cancer. Fam Cancer 2004; 3: 123-127.

18 Agostini M, Tibiletti MG, Lucci-Cordisco E et al: Two PMS2 mutations in a Turcot syndrome family with small bowel cancers. Am J Gastroenterol 2005; 100: 1886-1891.

19 Hegde MR, Chong B, Blazo ME et al: A homozygous mutation in MSH6 causes Turcot syndrome. Clin Cancer Res 2005; 11: $4689-4693$.

20 de la Chapelle A: Testing tumors for microsatellite instability. Eur J Hum Genet 1999; 7: 407-408.

21 Buhard O, Cattaneo F, Wong YF et al: Multipopulation analysis of polymorphisms in five mononucleotide repeats used to determine the microsatellite instability status of human tumors. J Clin Oncol 2006; 24: 241-251.

22 Pastrello C, Baglioni S, Tibiletti MG et al: Stability of BAT26 in tumours of hereditary nonpolyposis colorectal cancer patients with MSH2 intragenic deletion. Eur J Hum Genet 2006; 14: $63-68$.

23 Suraweera N, Duval A, Reperant M et al: Evaluation of tumor microsatellite instability using five quasimonomorphic mononucleotide repeats and pentaplex PCR. Gastroenterology 2002; 123: $1804-1811$.

24 Louis DN, Ohgaki H, Wiestler OD et al: The 2007 WHO classification of tumours of the central nervous system. Acta Neuropathol 2007; 114: 97-109. 
25 Coombs NJ, Gough AC, Primrose JN: Optimisation of DNA and RNA extraction from archival formalin-fixed tissue. Nucleic Acids Res 1999; 27: e12.

26 Clendenning $\mathrm{M}$, Hampel $\mathrm{H}$, LaJeunesse $\mathrm{J}$ et al: Long-range PCR facilitates the identification of PMS2-specific mutations. Hum Mutat 2006; 27: 490-495.

27 Wooster R, Cleton-Jansen AM, Collins N et al: Instability of short tandem repeats microsatellites in human cancers. Nat Genet 1994; 6: $152-156$

28 Mizoguchi $\mathrm{M}$, Inamura $\mathrm{T}$, Ikezaki $\mathrm{K}$ et al: Patient survival and microsatellite instability in gliomas by high-resolution fluorescent analysis. Oncol Rep 1999; 6: 791-795.

29 Martinez R, Schackert HK, Plaschke J, Baretton G, Appelt H, Schackert G: Molecular mechanisms associated with chromosomal and microsatellite instability in sporadic glioblastoma multiforme. Oncology 2004; 66: 395-403.

30 Wagner A, Reddinguis $\mathrm{R}$, Kros $\mathrm{J}$ et al: Wilms tumor and glioblastoma in a child with a double MLH1 germline mutation. Fam Cancer 2003; 1 (suppl.): 57.

31 Poley J-W, Wagner A, Hoogmans MCP et al: Biallelic germline mutations of mismatch-repair genes. Cancer 2007; 109: $2349-2356$.
32 Duval A, Reperant M, Compoint A et al: Target gene mutation profile differs between gastrointestinal and endometrial tumors with mismatch repair deficiency. Cancer Res 2002; 62: $1609-1612$.

33 Nakagawa H, Lockman JC, Frankel WL et al: Mismatch repair gene PMS2: disease-causing germline mutations are frequent in patients whose tumors stain negative for PMS2 protein, but paralogous genes obscure mutation detection and interpretation. Cancer Res 2004; 64: 4721-4727.

34 Gottschling S, Reinhard H, Pagenstecher C et al: Hypothesis: possible role of retinoic acid therapy in patients with biallelic mismatch repair gene defects. Eur J Pediatr 2008; 167: 225-229.

35 Scott RH, Homfray T, Huxter NL et al: Familial T-cell nonHodgkin lymphoma caused by biallelic MSH2 mutations. J Med Genet 2007; 44: e83.

36 Jackson CC, Holter S, Pollett A et al: Cafè-au-lait macules and pediatric malignancy caused by biallelic mutations in the DNA mismatch repair (MMR) gene PMS2. Pediatr Blood Cancer 2008; 50: $1268-1270$.

37 Tan TY, Orme LM, Lynch E et al: Biallelic PMS2 mutations and a distinctive childhood cancer syndrome. J Pediatr Hematol Oncol 2008; 30: 254-257. 